\title{
CATALOGO DE LA FLORA VASCULAR DEL PARQUE NACIONAL PALI AIKE, XII REGION, CHILE
}

\section{CHECKLIST OF THE VASCULAR FLORA OF PALI AIKE NATIONAL PARK, PATAGONIA, CHILE}

\author{
Erwin Domínguez ${ }^{1}$, Clodomiro Marticorena ${ }^{2},{\text { Arve Elvebakk }{ }^{3} \& \text { Aníbal Pauchard }}^{4}$ \\ ${ }^{1}$ Centro de Estudios del Cuaternario, Universidad de Magallanes, Casilla 113-D, Punta Arenas, Chile. \\ Email:erwindominguez925@hotmail.com \\ ${ }^{2}$ Departamento de Botánica, Universidad de Concepción, Casilla 160-C, Concepción, Chile. \\ ${ }^{3}$ Department of Biology, Faculty of Science, University of Troms, N-9037 Troms, Norway. \\ ${ }^{4}$ Facultad de Ciencias Forestales, Universidad de Concepción, Casilla 160-C, Concepción, Chile.
}

\begin{abstract}
We present a checklist of vascular plants for Pali Aike National Park, one of the world southernmost protected areas, located in Chilean Patagonia. The park conserves remnants of the Patagonian steppe ecosystem of the eastern side of the Straits of Magellan. 164 vascular species were recorded, from 44 families and 106 genera, comprising one Pteridophyta, one Gnetophyta, 114 Magnoliopsida and 48 Liliopsida. Exotic species make up 11\% of the flora.
\end{abstract}

\section{INTRODUCCION}

El Parque Nacional Pali Aike es uno de los más australes del mundo y la única unidad del Sistema Nacional de Areas Silvestres Protegidas de Chile en el cuál está representada la estepa patagónica austral. La estructura y composición florística de dicho ecosistema han sido muy alteradas producto de la explotación ganadera (Collantes et al. 1999). El parque fue creado en el año 1970 con una superficie original de 3.000 hectáreas para proteger hallazgos arqueológicos y geológicos (Massone 1981), siendo luego incrementada su superficie a 5.030 hectáreas en el año 1994 con el fin de conservar la flora y la fauna. Su aislamiento a través de un alambrado divisorio ha convertido al parque en una verdadera reserva, que ha favorecido la conservación in situ de la flora de un ecosistema semiárido amenazado y escasamente estudiado. Este trabajo entrega una primera lista de las plantas vasculares, tanto nativas como introducidas, más su forma de vida, la cual contribuirá con la formación de una base de datos, actualizable en forma permanente, que permitirá el monitoreo de los cambios en la diversidad florística y el estudio de la dinámica de su vegetación.
El Parque Nacional Pali Aike se ubica entre los $52^{\circ} 04^{\prime}$ y $52^{\circ} 05^{\prime}$ de latitud Sur y $69^{\circ} 47^{\prime}$ y $69^{\circ} 51^{\prime}$ de longitud Oeste, a lo largo de la frontera entre Chile y Argentina. El área de estudio corresponde a una extensa meseta de 110 a 250 msnm, donde destacan dos geoformas: la primera de origen volcánico, integrada por mares, conos y escoriales (Skewes 1978); la segunda de origen glacifluviales, integradas por morrenas marginales (Marangunic 1974, Clapperton et al. 1992). El clima corresponde al de Estepa Frío, con temperaturas medias entre 3 y $4^{\circ} \mathrm{C}$, caracterizado por fuertes vientos y precipitaciones medias anuales entre 200 y $250 \mathrm{~mm}$, distribuidas homogéneamente durante el año (Endlicher \& Santana 1988). La vegetación forma parte de la provincia biótica de la estepa patagónica, su fisonomía es dominada por pastizales y matorrales bajos.

Entre los años 2000 y 2004 se realizaron colectas intensivas en un total de 12 áreas del parque (Tabla I). Esta información fue complementada con la revisión de las colecciones de los herbarios del Instituto de la Patagonia (HIP) y de la Universidad de Concepción (CONC) (Holmgren et al. 1990). 
Los ejemplares colectados se identificaron siguiendo a Moore (1983), Correa (1969, 1971, 1978, 1984a, 1984b, 1988, 1999), Marticorena \& Rodríguez (1995), Matthei (1995), Peralta (1988), Ulibarri \& Burkart (2000), Domínguez \& Elvebakk (2002). Las especies fueron clasificadas de acuerdo a su origen geográfico en nativas o introducidas (Marticorena \& Quezada 1985, Matthei 1995, Henríquez et al. 1995), y de acuerdo a su forma de vida en: hierbas anuales, bienales, perennes, subarbustos y arbustos (Arroyo et al. 2000). Los ejemplares herborizados fueron depositados en el Herbario de la Universidad de Concepción (CONC) y sus duplicados fueron enviados al Herbario del Instituto de Botánica Darwinion (SI), Argentina.

TABLa I. Ubicación de zonas de colecta en el Parque Nacional Pali Aike, XII Región, Chile.

\begin{tabular}{ll}
\hline Sitios de colecta & Coordenadas \\
\hline Hito XVI & $52^{\circ} 03^{\prime} 56^{\prime \prime} \mathrm{S}, 69^{\circ} 48^{\prime} 87^{\prime \prime} \mathrm{O}$ \\
Laguna Ana & $52^{\circ} 04^{\prime} 53^{\prime \prime} \mathrm{S}, 69^{\circ} 47^{\prime} 17^{\prime \prime} \mathrm{O}$ \\
Laguna de los Cisnes & $52^{\circ} 04^{\prime} 60^{\prime \prime} \mathrm{S}, 69^{\circ} 46^{\prime} 39^{\prime \prime} \mathrm{O}$ \\
Laguna Seca & $52^{\circ} 06^{\prime} 59^{\prime \prime} \mathrm{S}, 69^{\circ} 46^{\prime} 52^{\prime \prime} \mathrm{O}$ \\
Cerro Pionero & $52^{\circ} 06^{\prime} 62^{\prime \prime} \mathrm{S}, 69^{\circ} 44^{\prime} 25^{\prime \prime} \mathrm{O}$ \\
Cueva de los Chingues & $52^{\circ} 05^{\prime} 97^{\prime \prime} \mathrm{S}, 69^{\circ} 45^{\prime} 07^{\prime \prime} \mathrm{O}$ \\
Cueva de Pali Aike & $52^{\circ} 06^{\prime} 59^{\prime \prime} \mathrm{S}, 69^{\circ} 42^{\prime} 43^{\prime \prime} \mathrm{O}$ \\
Escorial del Diablo & $52^{\circ} 06^{\prime} 67^{\prime \prime} \mathrm{S}, 69^{\circ} 41^{\prime} 24^{\prime \prime} \mathrm{O}$ \\
Mares internos & $52^{\circ} 05^{\prime} 77^{\prime \prime} \mathrm{S}, 69^{\circ} 43^{\prime} 61^{\prime \prime} \mathrm{O}$ \\
Morada del Diablo & $52^{\circ} 06^{\prime} 34^{\prime \prime} \mathrm{S}, 69^{\circ} 40^{\prime} 40^{\prime \prime} \mathrm{O}$ \\
Cerros Gemelos & $52^{\circ} 07^{\prime} 43^{\prime \prime} \mathrm{S}, 69^{\circ} 42^{\prime} 18^{\prime \prime} \mathrm{O}$ \\
Guardería & $52^{\circ} 07^{\prime} 49^{\prime \prime} \mathrm{S}, 69^{\circ} 46^{\prime} 00^{\prime \prime} \mathrm{O}$ \\
\end{tabular}

Un total de 164 especies, distribuidas en 44 familias y 106 géneros, fue colectado dentro del parque. Las especies nativas constituyeron el $89 \%$ de la flora (Tabla II). Las familias más numerosas son Poaceae con 34 especies, seguida por Asteraceae con 27. La composición taxonómica indicó una fuerte dominancia de Magnoliófitas, las que representan un $99 \%$ de la flora total (Tabla II). La única gnetófita encontrada fue Ephedra frustillata Miers (Ephedraceae). La forma de vida dominante en el parque fueron las herbáceas perennes $(79 \%$ de la flora: 119 nativas y 11 introducidas), en contraste con la escasa representación de plantas anuales (6\% de la flora: 6 nativas y 4 exóticas) (Tablas III). La presencia de aguadas, pozos antiguos de yacimientos de petróleo y caminos abandonados, son evidencias de los disturbios provocados por el pastoreo y la actividad petrolera, cuya consecuencia se manifiesta en la presencia de 18 especies introducidas ( $11 \%$ de la flora vascular del parque) (Tabla II). Entre las especies introducidas destaca la presencia de Hieracium pilosella L. (Asteraceae) en el sector Cerros Gemelos, primer hallazgo en un ambiente xérico. Esta hierba perenne, invasora de praderas húmedas, se ha convertido en una maleza problemática en la XII Región (Covacevich 2001). De acuerdo a las visitas realizadas desde el año 2000, la aparición de esta especie es reciente en el parque. Su presencia no tiene relación aparente con la actividad ganadera sino al parecer con la actividad turística, ya que los individuos encontrados se distribuyen en las bermas de los caminos por donde transitan los vehículos dentro del parque.

TABLA II. Composición taxonómica de la flora vascular del Parque Nacional Pali Aike, XII Región, Chile.

\begin{tabular}{lccccc}
\hline & Pteridophyta & Gnetophyta & Magnoliopsida & Liliopsida & \multicolumn{1}{c}{ Total } \\
\hline Nativas & $1(1 \%)$ & $1(1 \%)$ & $101(69 \%)$ & $43(29 \%)$ & $146(89 \%)$ \\
Introducidas & $0(0 \%)$ & $0(0 \%)$ & $13(72 \%)$ & $5(29 \%)$ & $18(11 \%)$ \\
Flora total & $1(1 \%)$ & $1(1 \%)$ & $114(70 \%)$ & $48(28 \%)$ & 164 \\
& & & & & \\
\hline
\end{tabular}


Flora vascular Parque Nacional Pali Aike: Domínguez, E. ET AL.

TABLA III. Formas de vida de la flora nativa e introducida del Parque Nacional Pali Aike, XII Región, Chile.

\begin{tabular}{lccccccc}
\hline & Anual & Bienal & $\begin{array}{c}\text { Anual- } \\
\text { Bienal }\end{array}$ & $\begin{array}{c}\text { Hierba } \\
\text { perenne }\end{array}$ & Subarbusto & Arbusto & Total \\
\hline Nativas & $6(4 \%)$ & $1(1 \%)$ & $0(0 \%)$ & $119(81 \%)$ & $16(11 \%)$ & $4(3 \%)$ & $146(89 \%)$ \\
Introducidas & $4(22 \%)$ & $0(0 \%)$ & $3(17 \%)$ & $11(61 \%)$ & $0(0 \%)$ & $0(0 \%)$ & $18(11 \%)$ \\
Flora total & $10(6 \%)$ & $1(1 \%)$ & $3(2 \%)$ & $130(79 \%)$ & $16(10 \%)$ & $4(2 \%)$ & 164 \\
\hline
\end{tabular}

\section{AGRADECIMIENTOS}

Agradecemos a María Negritto, Alicia Marticorena y al personal del Herbario de la Universidad de Concepción. A Emilio Ulibarri y al personal del herbario del Instituto de Botánica Darwinion, Argentina. También al profesor Nilo Covacevich y al Instituto de Investigaciones Agropecuarias, XII Región. Y al Sr. Miguel Gallardo de la Corporación Nacional Forestal, XII Región.

\section{BIBLIOGRAFIA}

Arroyo, M.T.K., O. Matthei, C. Marticorena, M. MuÑoz, F. PÉREZ \& A.M. HuMAÑa. 2000. La flora vascular de la Reserva Nacional Bellotos del Melado, VII Región, Chile: Un catálogo documentado. Gayana Botanica 57:117-139.

Collantes, M.B., J. Anchorena \&. A.M. Cingolani. 1999.

The steppes of Tierra del Fuego: Floristic and growth form patterns controlled by soil fertility and moisture. Plant Ecology 148:61-75.

Clapperton, C.M. 1992. La última glaciación y deglaciación en el Estrecho de Magallanes: Implicaciones para el poblamiento de Tierra del Fuego. Anales del Instituto de la Patagonia, Serie Ciencias Naturales 21: 113-128.

Correa, M.N. 1969. Flora Patagónica. Parte II. Typhaceae a Orchidaceae. Colección Científica del INTA. Tomo VIII. Buenos Aires. 219 pp.

Correa, M.N. 1971. Flora Patagónica. Parte VII. Compositae. Colección Científica del INTA. Tomo VIII. Buenos Aires. 451 pp.

Correa, M.N. 1978. Flora Patagónica. Parte III. Gramineae. Colección Científica del INTA. Tomo VIII. Buenos Aires. 563 pp.

Correa, M.N. 1984a. Flora Patagónica. Parte IVa. Dicotyledoneae: Dialipétalas (Salicaceae a Cruciferae) Colección Científica del INTA. Tomo VIII. Buenos Aires. 559 pp.
Correa, M.N. 1984b. Flora Patagónica. Parte IVb. Dicotyledoneae: Dialipétalas (Droseraceae a Leguminosae). Colección Científica del INTA. Tomo VIII. Buenos Aires. 309 pp.

Correa, M.N. 1988. Flora Patagónica. Parte V. Dicotyledoneae: Dialipétalas (Oxalidaceae a Cornaceae). Colección Científica del INTA. Tomo VIII. Buenos Aires. 381 pp.

Correa, M.N. 1999. Flora Patagónica. Parte VI. Dicotyledoneae: Gamopétalas (Ericaceae a Calyceraceae). Colección Científica del INTA. Tomo VIII. Buenos Aires. 517 pp.

Covacevich, C. 2001. Guía de manejo de coironales: Bases para el planeamiento de la estancia. Boletín INIA 47:1-23.

CRONQUIST, A. 1981. An integrated system of classification of flowering plants. Columbia University Press, New York. 1262 pp.

Domínguez, E. y A. Elvebakk (2002). Lecanophora subacaule Krapov. (Malvaceae), nuevo registro para la flora de Chile. Gayana Botanica 59: 43-47.

Endlicher, W. \& A. SANTANa. 1988. El clima del sur de la Patagonia y sus aspectos ecológicos. Un siglo de mediciones climatológicas en Punta Arenas. Anales Instituto de la Patagonia, Serie Ciencias Naturales 26: 119-128.

Henríquez, J.M., E. Pisano \& C. Marticorena. 1995. Catálogo de la flora vascular de Magallanes (XII Región), Chile. Anales del Instituto de la Patagonia. Serie Ciencias Naturales 23: 5-30.

Holmgren, P. K., Holmgren, N.H. \& L. C. Barnett. 1990. Index Herbariorum. Part I: The Herbaria of the World. New York Botanical Garden, New York. 693 pp.

Marangunic, C. 1974. Los depósitos glaciales de la pampa magallánica. Revista Geográfica de Chile "Terra Australis" 22-23: 5 - 11.

Marticorena, C. \& R. Rodríguez. 1995. Flora de Chile. Vol. 1. Ediciones Universidad de Concepción, Concepción, Chile. 351 pp.

Marticorena, C. \& M. QueZAdA. 1985. Catálogo de la flora vascular de Chile. Gayana Botanica 42: 1-157 pp.

Massone, M. 1981. Arqueología de la región volcánica de Pali Aike (Patagonia meridional chilena). Anales del 
Instituto de la Patagonia 12: 95-124.

Matther, O. 1995. Manual de las malezas que crecen en Chile. Alfabeta Impresores, Santiago, Chile. 547 pp.

Moore, D.M. 1983. Flora of Tierra del Fuego. Oswestry, Saint Louis, EE.UU. 369 pp.

Peralta, I.E. 1988. Sinopsis de las especies de Calandrinia (Portulacaceae) de los Andes Mendocinos. Boletín de la Sociedad Argentina de Botánica 25: 512-537.

SKEWES, M. 1978. Geología, petrología, química y origen de los volcanes del área de Pali Aike. Anales del Instituto de la Patagonia 9: 95-106.

Ulibarri, E. \& A. BurKart. 2000. Sinopsis de las especies de Adesmia (Leguminosae, Adesmieae) de la Argentina. Darwiniana 38: 59-126.

\section{FLORA DEL PARQUE NACIONAL PALI AIKE}

Listado de especies nativas e introducidas de Pali Aike, considerando el ciclo de vida y la forma biológica. $(\mathrm{A}=$ Hierba Anual; $\mathrm{B}=$ Hierba Bienal; $\mathrm{H}=$ Hierba Perenne; $\mathrm{S}=$ Subarbusto; $\mathrm{F}=$ Arbusto; I= Introducida).

\section{PTERIDOPHYTA}

Woodsiaceae

1. H Cyptopteris fragilis (L.) Bernh. var. apiiformis (Gand.) C.Chr.

\section{GNETOPHYTA}

Ephedraceae

\section{S Ephedra frustillata Miers}

\section{MAGNOLIOPHYTA}

Magnoliopsida (Dicotyledoneae)

Apiaceae

3. $\mathrm{H}$ Azorella fuegiana Speg.

4. S Azorella monantha Clos

5. S Azorella trifurcata (Gaertn.) Pers.

6. S Bolax gummifera (Lam.) Spreng.

7. H Huanaca acaulis Cav.

8. H Huanaca burkartii Mathias et Constance

9. H Osmorhiza berterii DC.

Asteraceae

10. H Antennaria chilensis J.Remy

11. A Artemisia magellanica Sch.Bip.

12. S Baccharis magellanica (Lam.) Pers.

13. F Chiliotrichum diffusum (G.Forst.)

Kuntze

14. H Erigeron myosotis Pers.

15. H Erigeron patagonicus Phil.

16. H Gamochaeta spiciformis (Sch.Bip.) Cabrera

17. S Gutierrezia baccharoides Sch.Bip.
18. H - I Hieracium pilosella L. subsp. euronotum Nägeli et Peter

19. H Hypochaeris incana (Hook. et Arn.) Macloskie var. incana

20. A - I Leuchanthemum vulgare Lam.

21. H Leucheria eriocephala Speg.

22. H Leucheria hahnii Franchet

23. H Leucheria purpurea (Vahl) Hook. et Arn.

24. H - I Matricaria discoidea DC.

25. S Nardophyllum bryoides (Lam.) Cabrera

26. H Nassauvia aculeata (Less.) Poepp. et Endl. var. azorelloides (Speg.) Cabrera

27. H Nassauvia darwinii (Hook et Arn.) O.Hoffm. et Dusén

28. H Perezia pilifera (D.Don.) Hook. et Arn.

29. H Perezia recurvata (Vahl) Less.

30. H Senecio alloeophyllus O.Hoffm.

31. H Senecio kingii Hook.f. var. kingii

32. H Senecio magellanicus Hook. et Arn.

33. H Senecio miser Hook.f.

34. F Senecio patagonicus Hook. et Arn. var. patagonicus

35. H Taraxacum gilliesii Hook. et Arn

36. H - I Taraxacum officinale Weber ex F.H.Wigg.

Berberidaceae

37. F Berberis microphylla G.Forst.

38. S Berberis empetrifolia Lam.

Boraginaceae

39. B Amsinckia calycina (Moris) Chater

40. H - I Myosotis stricta Link ex Roem. et Schult.

41. H Plagiobothrys calandrinioides (Phil.) I.M.Johnst.

Brassicaceae

42. AB - I Capsella bursa-pastoris (L.) Medik.

43. AB - I Descurainia sophia (L.) Webb ex Prantl

44. H Draba funiculosa Hook.f.

45. H Draba magellanica Lam.

46. A - I Draba verna L.

47. H Onuris papillosa O.E.Schulz

48. H Sisymbrium magellanicum (Pers.) Hook.f.

49. H Thlaspi magellanicum Comm. ex Poir.

Calyceraceae

50. H Boopis filifolia Speg.

51. H Boopis patagonica Speg.

Campanulaceae

52. H Lobelia oligophylla (Wedd.) Lammers

Caryophyllaceae

53. H Arenaria digyna D.F.K.Schltdl.

54. H - I Cerastium arvense L.

55. H Colobanthus lycopodioides Griseb.

56. H Colobanthus subulatus (d'Urv.) Hook.f.

57. H Colobanthus quitensis (Kunth) Bartl. 
58. H Silene magellanica (Desr.) Bocquet

59. H Stellaria debilis d'Urv.

Chenopodiaceae

60. H Chenopodium carnosulum Moq.

61. A Suaeda patagonica Speg.

Empetraceae

62. S Empetrum rubrum Vahl ex Willd.

Ericaceae

63. S Gaultheria pumila (L.f.) D.J.Middleton var. leucocarpa (DC.) D.J.Middleton

Euphorbiaceae

64. H Euphorbia collina Phil. var. minor (Hicken) Subils

Fabaceae

65. F Adesmia boronioides Hook.f.

66. H Adesmia lotoides Hook.f.

67. H Adesmia pumila Hook.f.

68. S Adesmia salicornioides Speg.

69. $\mathrm{H}$ Adesmia villosa Hook.f.

70. H Astragalus nivicola Gómez-Sosa

71. H Astragalus palenae (Phil.) Reiche var. palenae

72. H Vicia bijuga Gillies ex Hook. et Arn.

Gentianaceae

73. A Gentianella magellanica (Gaudich.)

Fabris ex D.M.Moore

74. A Gentiana sedifolia Kunth

Geraniaceae

75. AB - I Erodium cicutarium L'Hér. ex Aiton

76. H Geranium magellanicum Hook.f.

Hydrophyllaceae

77. H Phacelia secunda J.F.Gmel.

Lamiaceae

78. S Satureja darwinii (Benth.) Briq.

79. H Scutellaria nummulariifolia Hook.f.

Malvaceae

80. H Lecanophora subacaule Krapov.

Oxalidaceae

81. H Oxalis enneaphylla Cav. subsp. ibari (Phil.) Lourteig

82. H Oxalis squamoso-radicosa Steud.

83. H Oxalis loricata Dusén

Plantaginaceae

84. H Plantago barbata G.Forst. subsp. barbata

85. H Plantago maritima L.

Plumbaginaceae

86. H Armeria maritima (Mill.) Willd.
Polemoniaceae

87. A Microsteris gracilis (Hook.) Greene

88. H Polemonium micranthum Benth.

Polygalaceae

89. H - I Rumex acetosella L.

Portulacaceae

90. H Calandrinia caespitosa Gillies ex Arn

Primulaceae

91. A Androsace salasii Kurtz

92. H Primula magellanica Lehm.

Ranunculaceae

93. H Anemone multifida Poir.

94. H Hamadryas delfinii Phil. ex Reiche

95. H Myosurus patagonicus Speg.

96. H Ranunculus aquatilis L.

97. H - I Ranunculus repens L.

98. H Ranunculus pseudotrullifolius Skottsb.

Rhamnaceae

99. S Discaria chacaye (G.Don) Tortosa

Rosaceae

100. H Acaena caespitosa Gillies ex Hook. et Arn.

101. S Acaena integerrima Gillies ex Hook. et Arn.

102. S Acaena magellanica (Lam.) Vahl

103. H Acaena pinnatifida Ruiz et Pav.

104. H Acaena poeppigiana Gay

105. H Geum magellanicum Pers.

Rubiaceae

106. A - I Galium aparine L.

107. H Galium araucanum Phil.

108. H Oreopolus glacialis (Poepp.) Ricardi

Santalaceae

109. H Arjona patagonica Hombr. et Jacquinot

110. H Arjona pusilla Hook.f.

Saxifragaceae

111. H Saxifraga magellanica Poir.

Scrophylariaceae

112. H Calceolaria uniflora Lam.

Solanaceae

113. H Jaborosa magellanica (Griseb.) Phil.

Valeraniaceae

114. H Valeriana carnosa $\mathrm{Sm}$.

Verbenaceae

115. S Junellia odonellii Moldenke

Violaceae 
Gayana Bot. 61(2), 2004

116. H Viola maculata Cav. var. maculata Liliopsida (Monocotyledoneae)

Alstroemeriaceae

117. H Alstroemeria patagonica Phil.

Cyperaceae

118. H Carex austroamericana G.A.Wheeler

119. H Carex macrosolen Steud.

120. H Carex sorianoi Barros

121. H Carex gayana E.Desv. var. gayana

122. H Eleocharis albibracteata Nees et Meyen ex Kunth

123. H Uncinia macrolepis Decne.

124. H Amphiscirpus nevadensis (S.Watson) Oteng-Yeb.

Iridaceae

125. H Olsynium biflorum (Thunb.) Goldblatt

126. H Sisyrinchium patagonicum Phil. ex Baker

Juncaceae

127. H Juncus arcticus Willd. var. mexicanus (Willd. ex Schult. et Schult.f.) Balslev

128. H Juncus scheuchzerioides Gaudich.

129. H Luzula alopecurus Desv.

130. H Luzula racemosa Desv.

Juncaginaceae

131. H Triglochin concinna Burtt Davy

Poaceae

132. H Agrostis inconspicua Kunze ex E.Desv.

133. H Agrostis glabra (J.Presl) Kunth

134. A - I Aira praecox L.

135. H Alopecurus magellanicus Lam. var. magellanicus

136. H Hierochloe pusilla Hack.

137. H Bromus catharticus Vahl

138. H Bromus setifolius J.Presl var. setifolius

139. H Calamagrostis stricta (Timm) Koeler

140. H Deschampsia antarctica E.Desv.

141. H Deschampsia flexuosa (L.) Trin.

142. H Deschampsia patula (Phil.) Pilg.

143. H Elymus magellanicus (E.Desv.) A.Löve

144. H Festuca gracillima Hook.f.

145. H Festuca magellanica Lam.

146. H Festuca pallescens (St.-Yves) Parodi

147. H Festuca pyrogea Speg.

148. H Hordeum comosum J.Presl

149. H Hordeum lechleri (Steud.) M.Schenck

150. H Hordeum patagonicum (Hauman)

Covas subsp. santacrucense (Parodi et Nicora) Bothmer, Giles et N.Jacobsen

151. H Jarava chrysophylla (E.Desv.) Peñail.

152. H Jarava humilis (Cav.) Peñail.

153. H Jarava ibarii (Phil.) Peñail.

154. $\mathrm{H}$ Phleum alpinum $\mathrm{L}$.

155. H - I Poa annua L.

156. H Poa alopecurus (Gaudich.) Kunth

157. H Poa alopecurus (Gaudich.) Kunth subsp. fuegiana (Hook.f) D.M.Moore et Dogg

158. H - I Poa compressa L.

159. H - I Poa nemoralis L.

160. H - I Poa pratensis L.

161. H Poa spiciformis (Steud.) Hauman et Parodi var. ibarii (Phil.) Giussani

162. H Puccinellia magellanica (Hook.f.) Parodi

163. H Rytidosperma virescens (E.Desv.) Nicora

164. H Trisetum cumingii (Nees ex Steud.) Parodi ex Nicora 